\title{
Specific Detection of Viable E.coli 0157:H7 Cells by Coupling Propidium Monoazide with Loop-Mediated Isothermal Amplification
}

\author{
Xihong Zhao, Jun Wang, Joong-Hyun Park, Fereidoun Forghani, Myoungsu Parkand Deog-Hwan Oh* \\ Department of Food Science and Biotechnology and Institute of Bioscience and Biotechnology, Kangwon National University, \\ Chuncheon, Gangwon 200-701, South Korea \\ *Corresponding author's e-mail: deoghwa@kangwon.ac.kr
}

\begin{abstract}
Traditional molecular detection methods cannot distinguish Escherichia coli O157:H7 in viable or dead state. In this study, the loop-mediated is other mal amplification (LAMP) method combined with propidium monoazide (PMA) treatment was developed to selectively detect the viable Escherichia coli O157:H7, but not dead cells. Four primers, including outer primers and inner primers, were specially designed for recognizing six distinct sequences on the species-specific $r f b E$ gene of Escherichia coli O157:H7 genome. PMA penetrated selectively through the compromised cell membranes and intercalated into DNA, amplification of DNA from dead cells was inhibited completely by $3.0 \mu \mathrm{g} / \mathrm{mL}$ PMA, whereas the DNA derived from viable cells was amplified remarkably within $1 \mathrm{~h}$ by PMA-LAMP. This study offers a novel molecular detection method to distinguish between the viable and dead cells.
\end{abstract}

Key words: E.coli O157:H7, propidium monoazide, rapid detection, viable cells. 